\title{
Characterization of Cobalt Sulfide Catalysts
}

\author{
Steven Rozeveld ${ }^{1}$, Wen-Sheng Lee ${ }^{1}$ and Paolo Longo ${ }^{2}$ \\ ${ }^{1}$ Dow Chemical Co., Midland, Michigan, United States, ${ }^{2}$ Gatan Inc, Pleasanton, California, United States
}

Transition metal sulfides are an important class of materials known for their structural variety along with technologically significant electronic and catalytic properties [1]. Cobalt sulfides have been investigated as oxygen-reduction catalyst and have the highest activity among all chalcogenides of nonprecious metals [2-3]. However, the phase diagram of cobalt sulfide is relatively complex containing several phases including $\mathrm{Co}_{4} \mathrm{~S}_{3}, \mathrm{Co}_{9} \mathrm{~S}_{8}, \mathrm{CoS}, \mathrm{Co}_{3} \mathrm{~S}_{4}, \mathrm{Co}_{2} \mathrm{~S}_{3}$, and $\mathrm{CoS}_{2}$ phases making analysis of samples with mixed cobalt sulfides phases challenging [1].

A commercial cobalt-sulfide catalyst was investigated in this study using scanning transmission electron microscopy (STEM) and energy dispersive x-ray spectroscopy (EDS) techniques to understand the material structure. The scanning-TEM image in Figure 1a shows the as-received nanoparticle $\mathrm{CoS}_{2}$ catalyst with a size ranging from 50-300nm. The EDS map showed a partial oxide surface coverage of the cobalt-sulfide particles (Figure 1b). EDS maps were collected at $80 \mathrm{keV}$ to improve the EDS signal and reduce beam damage.

Elemental mapping by EDS is less sensitive to variations in the sample thickness compared to electron energy loss (EELS) methods but the time required for data collection can be significant for EDS analysis of light elements. Improvements in the large detector solid angle in the Titan ChemiSTEM provides a significant improvement in this respect over conventional FEG-TEM instruments.

The catalyst uniformity was also studied using a dedicated PEELS system (Gatan Enfinium). SpectrumImage (SI) data sets were collected in a few minutes, similar to the EDS data collection time, but with a reduced probe current of $\sim 100 \mathrm{pA}$ vs $\sim 500 \mathrm{pA}$ for EDS. Using Dual-PEELS, low-loss and core-loss SI data were collected simultaneously from the catalyst regions (Figure 2a). Low loss SI map, shown in Figure $2 \mathrm{~b}$, indicated a relatively thickness range of 0-3.0 for this region. Multiple linear least squares (MLLS) fitting was used to identify the oxide phase distribution after deconvolution to remove plural scattering from the core-loss SI data (Figure 2c) [4]. PEELS analysis of the oxide phase (Figure 2d) showed that it contained sulfur with $\mathrm{S}-\mathrm{L}_{2,3}$ near-edge features consistent with sulfate coordination [5]. The near-edge fine structure of S-L2,3 edge was found to be useful as a fingerprint for identification of the sulfide and sulfate phase distribution.

Experimental: The TEM analysis was conducted at Dow Chemical using a ThermoScientific Titan Themis G2 80-300kV aberration probe corrected S/TEM which was equipped with ChemiSTEM EDS detectors and a Gatan Enfinium PEELS. Samples were prepared from a mixture of catalyst and methanol which was dispersed onto a standard lacey-carbon supported $\mathrm{Cu}$ TEM grid. 


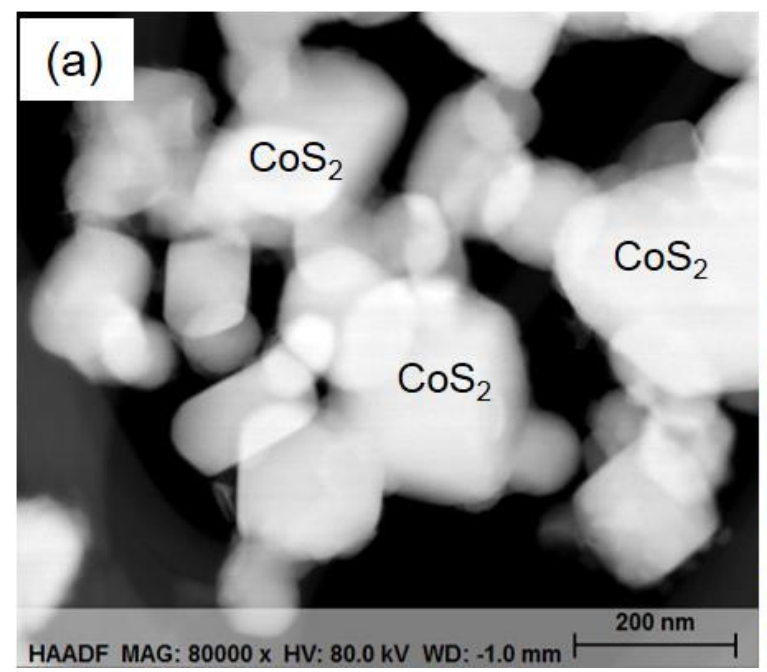

Figure 1. (a) Scanning TEM image 80keV oxygen, and sulfur. STEM Magnification $80 \mathrm{kx}$ / pixel, $512 \times 512,13 \mu \mathrm{sec}$ dwell time/ pixel/frame, 750 seconds total collection time.
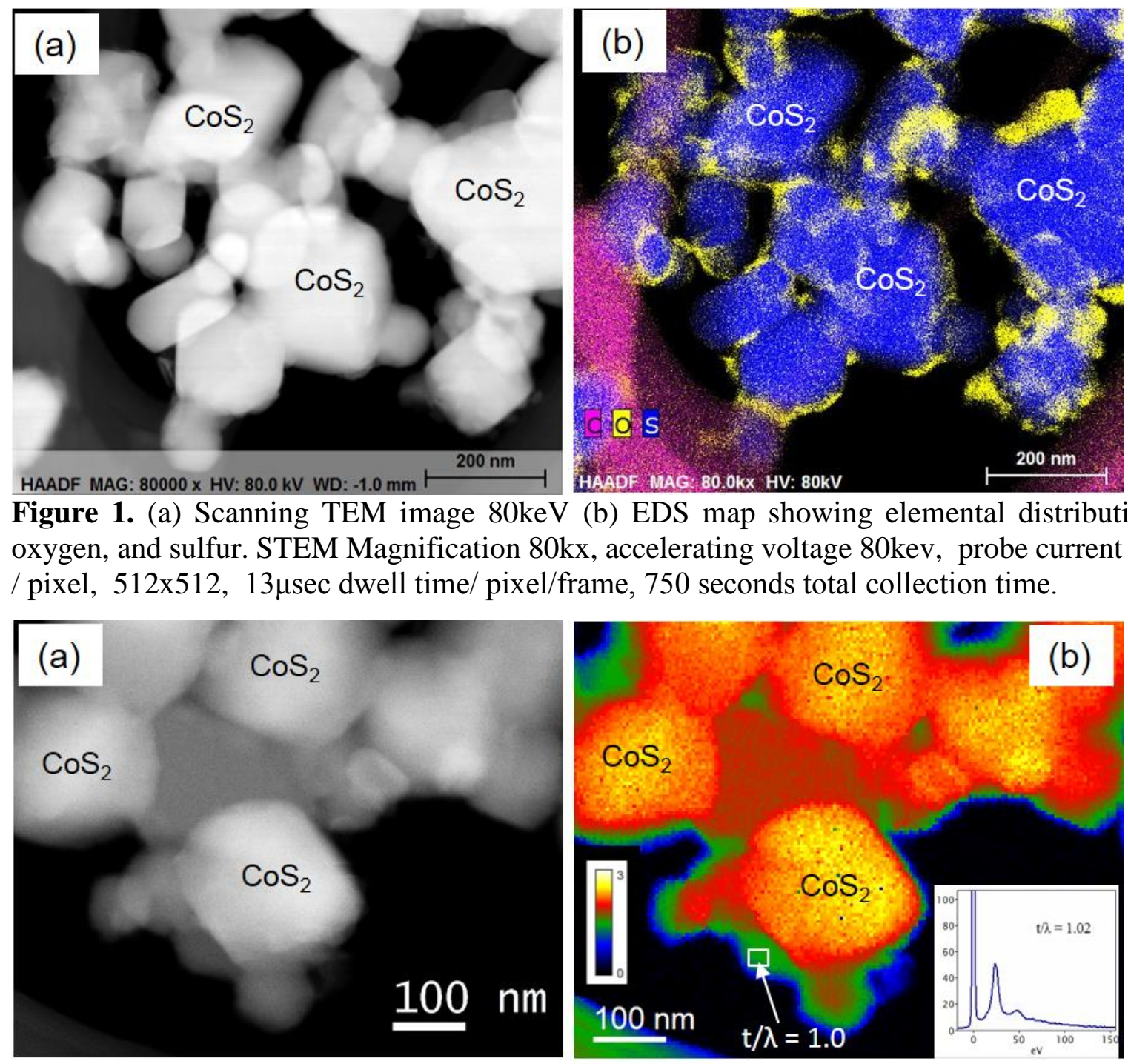

(b) EDS map showing elemental distribution of cobalt ,
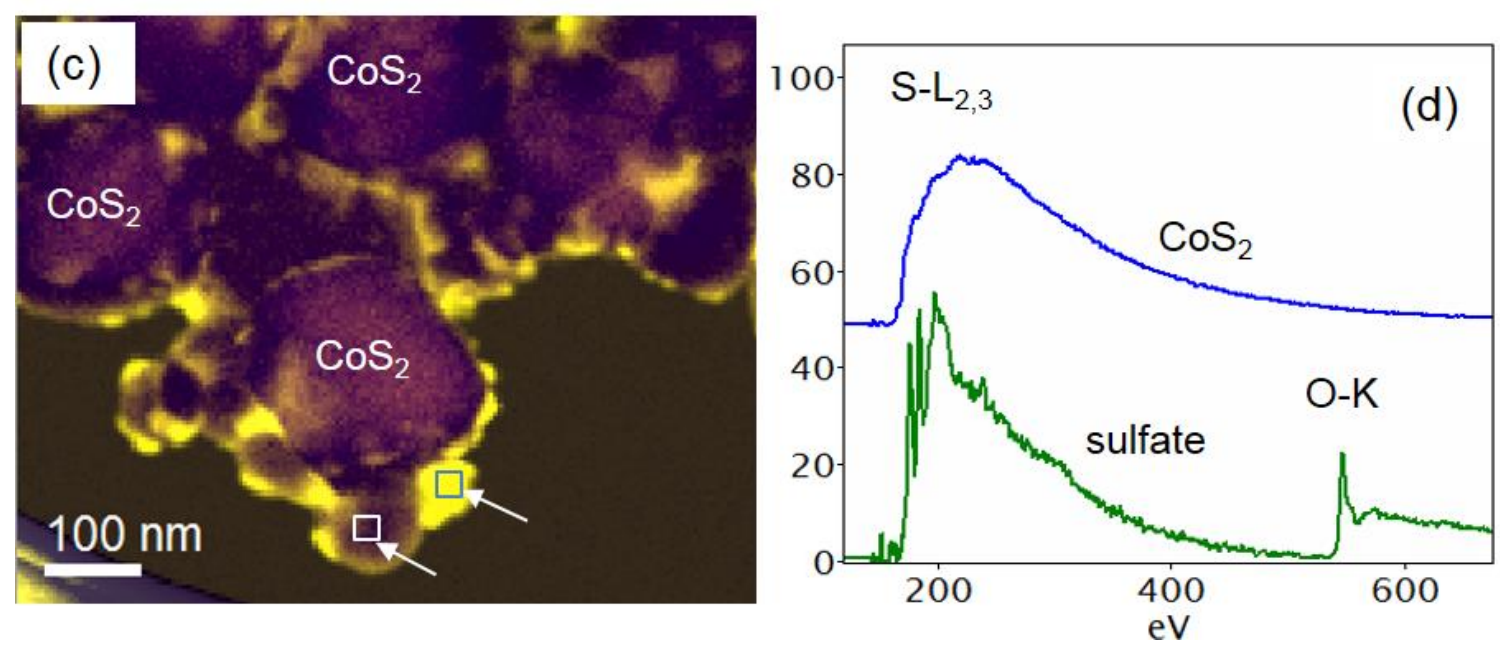

Figure 2. (a) scanning-TEM of CoS2 catalyst (b) relative thickness determined from low-loss SI (c) coreloss SI showing oxide phase distribution (d) overlay of background subtracted PEELS spectra from oxide 
and sulfide phases. SI $80 \mathrm{keV}, 4.5 \mathrm{~nm} /$ pixel, probe current $\sim 100 \mathrm{pA}, 168 \mathrm{x} 134$ pixels, low loss $\sim 100 \mu \mathrm{sec} / \mathrm{pixel}$, core-loss $\sim 30 \mathrm{msec} / \mathrm{pixel}, 0.4 \mathrm{eV} /$ channel, bin $=[2 \mathrm{x}, 26 \mathrm{x}]$.

\section{References}

1. Kumar, N. Raman, and A. Sundaresan, "Synthesis and Properties of Cobalt Sulfide Phases: $\mathrm{CoS}_{2}$ and Co9S8" Z. Anorg. Allg. Chem. 2014, 640, (6), 1069-1074

2. Wang, Y.Liang, Y. Li, and H. Dai, " Co1-x S-Graphene Hybrid: A High-Performance Metal Chalcogenide Electrocatalyst for Oxygen Reduction” Angew. Chem. Int. Ed. 2011, 50, 10969 -10972 3. Ganesan, M. Prabu, J. Sanetuntikul, and S. Shanmugam, "Cobalt Sulfide Nanoparticles Grown on Nitrogen and Sulfur Codoped Graphene Oxide: An Efficient Electrocatalyst for Oxygen Reduction and Evolution Reactions" ACS Catal. 2015, 5, 3625-3637

4. Bobynko, I. MacLaren, A. J. Craven "Spectrum imaging of complex nanostructures using DualEELS: I. digital extraction replicas" Ultramicroscopy 149 (2015) 9-20 Analytical TEM

5. Hofer and P. Golob, "New Examples for Near-Edge Fine Structures in Electron Energy Loss Spectroscopy" Ultramicroscopy 21 (1987) 379-384 379 